\title{
EBUS-centred versus EUS-centred mediastinal staging in lung cancer: a randomised controlled trial
}

\author{
Hyo Jae Kang, ${ }^{1}$ Bin Hwangbo, ${ }^{1}$ Geon-Kook Lee, ${ }^{2}$ Byung-Ho Nam, ${ }^{3}$ Hyun-Sung Lee, ${ }^{1}$ \\ Moon Soo Kim, ${ }^{1}$ Jong Mog Lee, ${ }^{1}$ Jae III Zo, ${ }^{1}$ Hee Seok Lee, ${ }^{1}$ Ji-Youn Han ${ }^{1}$
}

\begin{abstract}
- Additional material is published online only. To view please visit the journal online (http://dx.doi.org/10.1136/ thoraxjnl-2013-203881).

${ }^{1}$ Center for Lung Cancer, Research Institute and Hospital, National Cancer Center, Goyang, Korea ${ }^{2}$ Department of Pathology, Research Institute and Hospital, National Cancer Center, Goyang, Korea ${ }^{3}$ Cancer Biostatistics Branch, Research Institute for National Cancer Control and Evaluation, National Cancer Center, Goyang, Korea
\end{abstract}

\section{Correspondence to} Dr Bin Hwangbo,

Center for Lung Cancer, National Cancer Center, 323 Ilsan-ro, Ilsandong-gu, Goyang 410-769, Korea;

hbb@ncc.re.kr

Received 14 May 2013 Revised 21 September 2013 Accepted 7 October 2013 Published Online First 30 October 2013
To cite: Kang $\mathrm{HJ}$, Hwangbo B, Lee G-K, et al. Thorax 2014;69:261-268.
ABSTRACT
Background The impact of procedure sequence and primary procedure has not been studied in the combined application of endobronchial ultrasound-guided transbronchial needle aspiration (EBUS-TBNA) and endoscopic ultrasound-guided fine needle aspiration (EUS-FNA) in lung cancer staging.

Methods In a randomised controlled trial, 160 patients with histologically confirmed or strongly suspected potentially operable non-small cell lung cancer were enrolled (Group A, $n=80$, EBUS-centred; Group $B, n=80$, EUS-centred). EBUS-TBNA and EUS-FNA with an ultrasound bronchoscope were used as the first procedures in Groups A and B, respectively, and secondary procedures (EUS-FNA in Group A, EBUS-TBNA in Group B) were added.

Results Diagnostic values were evaluated in 148 patients (74 in each group). In Groups A and B the diagnostic accuracy $(93.2 \%(95 \% \mathrm{Cl} 87.5 \%$ to $99.0 \%)$ vs $97.3 \%(95 \% \mathrm{Cl} 93.6 \%$ to $101.0 \%), p=0.245)$ and sensitivity $(85.3 \%$ (95\% Cl $68.9 \%$ to $95.0 \%)$ vs $92.0 \%$ (95\% Cl $74.0 \%$ to $99.0 \%), p=0.431)$ in detecting mediastinal metastasis were not statistically different. In Group A, adding EUS-FNA to EBUS-TBNA did not significantly increase the accuracy (from $91.9 \%$ to $93.2 \%, p=0.754$ ) or sensitivity (from $82.4 \%$ to $85.3 \%$, $\mathrm{p}=0.742$ ). In group $\mathrm{B}$, adding EBUS-TBNA to EUS-FNA increased the accuracy (from $86.5 \%$ to $97.3 \%$, $\mathrm{p}=0.016$ ) and sensitivity (from $60.0 \%$ to $92.0 \%$, $p=0.008$ ). There were no intergroup differences in procedure time, cardiorespiratory parameters during procedures, complications or patient satisfaction. Conclusions Using a combination of EBUS-TBNA and EUS-FNA in mediastinal staging, we found that diagnostic values and patient satisfaction were not different between the EBUS-centred and EUS-centred groups. However, the necessity for EBUS-TBNA following EUS suggests that EBUS-TBNA is a better primary procedure in endoscopic mediastinal staging of potentially operable lung cancer.

Trial Registration number ClinicalTrials.gov number NCT01385111.

\section{INTRODUCTION}

Endobronchial ultrasound-guided fine needle aspiration (EBUS-TBNA) and endoscopic ultrasoundguided fine needle aspiration (EUS-FNA) are minimally invasive endoscopic methods for mediastinal staging of lung cancer. ${ }^{1-4}$ Endoscopic staging is recommended over surgical staging as the best first test for invasive mediastinal staging. ${ }^{1}$ Diagnostic yields of EBUS-TBNA or combined EBUS and EUS procedures

\section{Key messages}

What is the key question?

- Are there any differences according to procedure sequence and primary procedure in the combined application of endobronchial ultrasound-guided transbronchial needle aspiration (EBUS-TBNA) and endoscopic ultrasound-guided fine needle aspiration (EUS-FNA) in lung cancer staging?

What is the bottom line?

- In this study, diagnostic values and patient satisfaction were not different between the EBUS-centred and EUS-centred groups in mediastinal staging of potentially operable lung cancer. Additional diagnostic gain using EUS in the EBUS-centred group was minimal. However, a significant increase in diagnostic benefit was observed with the addition of EBUS in the EUS-centred group.

\section{Why read on?}

- Advantages of combined application of EBUS and EUS in lung cancer staging have been reported. However, studies on selection of the primary procedure and the effect of procedure sequence have not been performed. This study suggests that EBUS-TBNA is preferable as the primary procedure in endoscopic mediastinal staging of lung cancer.

were reported to be comparable to conventional mediastinoscopy. ${ }^{56}$

EBUS-TBNA and EUS-FNA are complementary methods in mediastinal staging because of different accessibility to the mediastinum. ${ }^{78}$ Several studies have shown advantages of combined endoscopic methods with EBUS and EUS in the staging of lung cancer. $^{9-13}$ Wallace et al $^{11}$ reported that combined EBUS-TBNA and EUS-FNA had a sensitivity of $93 \%$ in detecting malignant nodes in patients with suspected lung cancer, which was higher than either method alone. Herth et $a l^{12}$ also showed the complementary roles of EBUS and EUS in lung cancer staging. In our previous study we evaluated the additional roles of EUS following EBUS-TBNA in mediastinal staging of potentially operable lung cancer. The sensitivity in detecting mediastinal metastasis increased from $84.4 \%$ to $91.1 \%$ with 
the addition of the EUS procedure to EBUS-TBNA, although the difference was not statistically significant. Our study showed that accessibility to mediastinal nodal stations increased by adding EUS to EBUS-TBNA. ${ }^{13}$

It has not yet been determined which procedure should be the primary procedure in the combined approach of EBUS and EUS in mediastinal staging. Procedure sequence may affect the need for a secondary procedure and patient satisfaction. EBUS-TBNA has higher accessibility to the mediastinum in operable lung cancer, which may indicate that performing EBUS-TBNA prior to EUS is preferable. ${ }^{13}$ However, the EUS procedure is generally better tolerated than EBUS-TBNA. ${ }^{14} 15$ An EUS-dominant procedure may increase patient satisfaction.

We performed this study to investigate the impact of the primary procedure and procedure sequence on diagnostic values and procedure-related parameters in the combination of EBUS and EUS in mediastinal staging of lung cancer. EUS-FNA was performed with an ultrasound bronchoscope based on feasibility and safety shown in the literature. ${ }^{12-15}$ In this randomised controlled study, an EBUS-centred group was compared with an EUS-centred group; we expected that the two groups would show similar diagnostic accuracy. We compared diagnostic values, change of diagnostic values by adding secondary procedures and procedure-related parameters such as cardiorespiratory parameters, complications and satisfaction scores between groups.

\section{METHODS}

\section{Patients and study design}

This randomised parallel controlled trial was performed at the National Cancer Center in Goyang, Korea.

We enrolled patients with histologically confirmed or strongly suspected, potentially operable non-small cell lung cancer (NSCLC). Eligibility criteria are listed in box 1. Patents were

\section{Box 1 Eligibility criteria for this study*}

Inclusion criteria Histologically confirmed or strongly suspected non-small cell lung cancer

Potentially operable patients

Age 18-80 years

Exclusion criteria:

M1 diseaset

Inoperable T4 diseaset

Mediastinal infiltration or extranodal invasion of the mediastinal lymph node visible on chest CT

Confirmed supraclavicular lymph node metastasis

Pancoast tumours

Ground glass-dominant ( $>50 \%$ in diameter) $\mathrm{T} 1$ nodule $(\leq 3 \mathrm{~cm})$

Inoperable patients (after evaluating medical and surgical operability)

Patients who refused surgical treatment

Contraindications for bronchoscopy and oesophageal endoscopy

Drug reaction to lidocaine, midazolam, fentanyl

Pregnancy

${ }^{*}$ After staging work-up for non-small cell lung cancer with chest CT including upper abdomen, integrated PET-CT and brain imaging.

†Based on the international system for staging lung cancer. $^{28}$ randomly allocated (1:1) to Group A (EBUS-centred) and Group B (EUS-centred). Randomisation was done using computer-generated random block size permutation procedures; the block sizes used were 2, 4 and 6 . The randomisation was performed by an independent person and the sequence of the randomisation was sealed in envelopes. A stratification factor was the location of the primary tumours (right or left side). Patients were not informed about their allocated groups.

\section{Procedures and measurements}

EBUS-TBNA and EUS-FNA were performed using an ultrasound bronchoscope and a dedicated ultrasound processor (convex probe-EBUS; BF-UC260F-OL8 and EU-C2000; Olympus, Tokyo, Japan). Needle aspiration was performed with a 22-gauge needle (NA-201SX-4022; Olympus). Transbronchial and transoesophageal procedures were performed during a single session by the same bronchoscopist $(\mathrm{BH})$.

The procedures were performed under conscious sedation with patients in the supine position. Five $\mathrm{mL} \mathrm{5 \%} \mathrm{lidocaine} \mathrm{was}$ applied to the pharynx and larynx using a spray before the procedures. Midazolam $(2 \mathrm{mg})$ and fentanyl $(50 \mu \mathrm{g})$ were given intravenously immediately before the procedures. Additional doses of lidocaine, midazolam and fentanyl were added during the procedures at the bronchoscopist's discretion. Initially, $3 \mathrm{~L} / \mathrm{min}$ oxygen was administered via a nasal prong and the oxygen flow was increased when hypoxia occurred.

Conventional bronchoscopy was routinely performed in the same bronchoscopy session before the ultrasound procedures. In Group A, EBUS was performed first. After full inspection of the mediastinal nodes visible by EBUS, EBUS-TBNA was performed on nodal stations selected according to the judgement of the bronchoscopist. Following EBUS, EUS-FNA was performed as a secondary procedure on mediastinal nodes inaccessible or difficult to access by EBUS-TBNA. In Group B, EUS-FNA was performed first on selected nodes. EBUS-TBNA was added as a secondary procedure for inaccessible locations or for areas difficult to access by EUS-FNA.

Measured parameters including procedure time, number of lymph node stations, number of aspirations, amount of medications, cardiorespiratory parameters (blood pressure, heart rate, oxygen saturation $\left(\mathrm{SaO}_{2}\right)$, coughs and hypoxic event during the procedure), irritable movement, complications and procedure tolerance parameters (procedure difficulty and patient satisfaction) are described in online supplementary table 1. Procedure difficulty was assessed by the bronchoscopist and a main attendant nurse with a $10-\mathrm{cm}$ visual analog scale (VAS) immediately after procedures. Patient satisfaction parameters (procedure tolerance and willingness to repeat) were measured 2-4 hrs and 3-5 days after the procedure. Patient assessment of overall tolerance of the procedure and four sensations (cough, dyspnea, pain and nausea during the procedure), were collected with 10-cm VASs.

Cytopathological examinations were performed with EBUS-TBNA and EUS-FNA samples separately by a pathologist (GKL). We recommended open thoracotomy or video-assisted thoracic surgery with systematic lymph node dissection to patients whose endoscopic staging results did not show mediastinal metastases.

\section{Study end points}

The diagnostic standard for a malignant result was the pathological confirmation of malignancy by any tissue sampling (EBUS-TBNA, EUS-FNA or surgical biopsy). The diagnostic standard for a benign result was the surgical confirmation of 


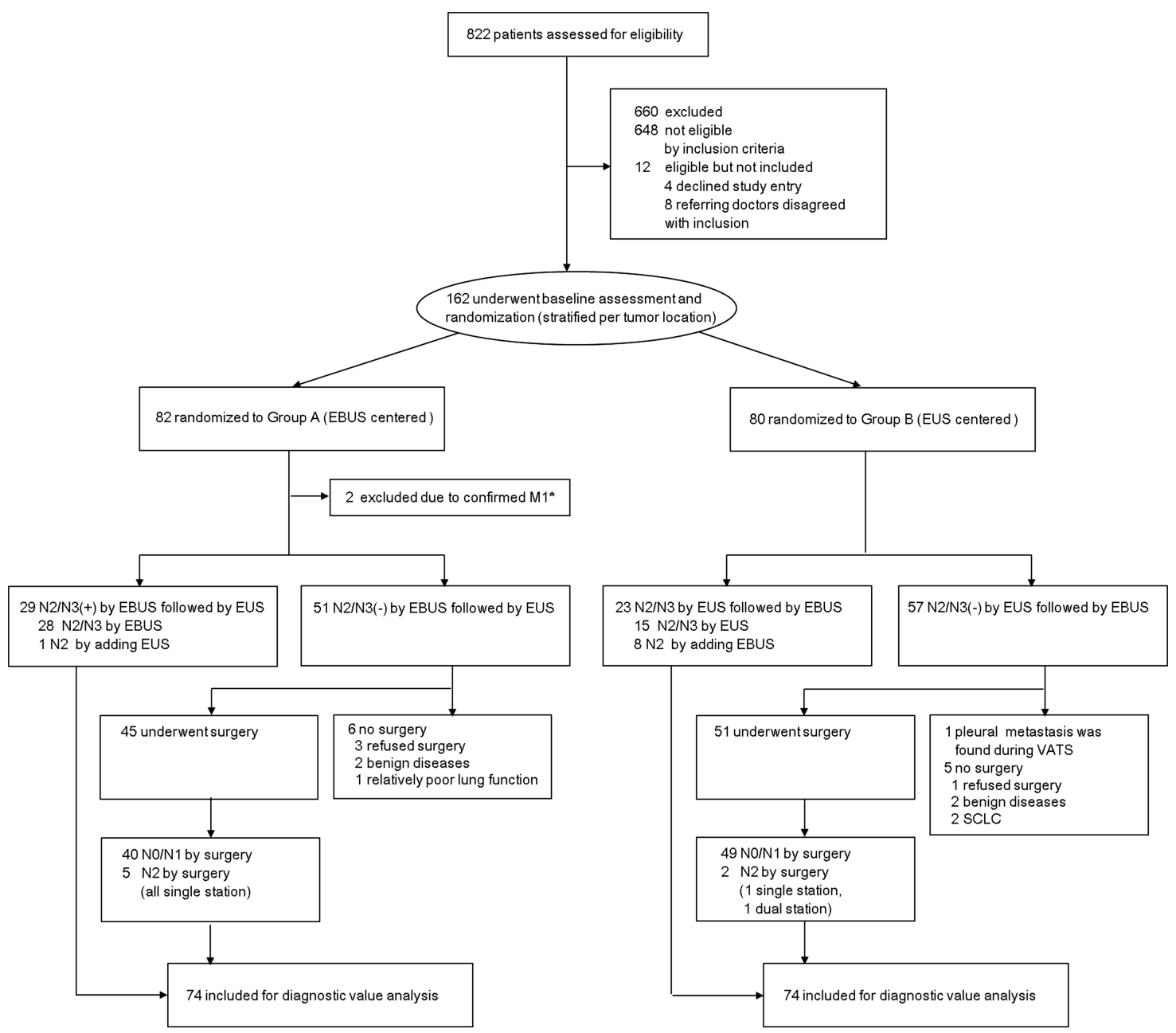

Figure 1 Enrolment and clinical course of study patients. *In two patients, distant metastases were diagnosed by bone MRI and reinterpretation of positron emission tomography (PET)/CT, respectively. EBUS, endobronchial ultrasound; EUS, endoscopic ultrasound; SCLC, small cell lung cancer; VATS, video-assisted thoracic surgery.

lesions showing no malignancy. The diagnostic accuracy, sensitivity and negative predictive value (NPV) for the detection of mediastinal metastasis (N2 or N3) were calculated using the standard definitions. The primary endpoint was diagnostic accuracy in detecting mediastinal metastasis. The sensitivity and NPV in detecting mediastinal metastasis were evaluated as secondary endpoints. Diagnostic values of primary procedures and additional diagnostic benefits of secondary procedures were assessed. Other secondary endpoints were procedure time, number of nodal stations and aspirations, amount of medications, cardiorespiratory parameters, number of patients with irritable movements during procedures, complications and procedure tolerance parameters.

\section{Statistical analysis}

Based on previous studies, ${ }^{6} 13$ the expected diagnostic accuracy of Group A was 95\%. The sample size calculation was based on the precision for estimating the accuracy of Group B. ${ }^{16}$ The expected accuracy of Group B would be the same as that of Group A. With a margin of error of 5\% and a follow-up loss of $10 \%$, the number of samples needed was 162 . Diagnostic values (accuracy, sensitivity and NPV), increments of diagnostic values and additional diagnostic benefit by secondary procedures were compared using a $Z$ test. $\chi^{2}$ or Fisher exact tests were used for evaluating categorical data (gender, final diagnosis, location of tumours, clinical staging, prevalence of mediastinal metastasis, hypoxic event, irritable movement, complications and unwillingness to repeat) as appropriate. Continuous variables (age, forced expiratory volume in $1 \mathrm{~s}\left(\mathrm{FEV}_{1}\right)$, blood pressure, heart rate, $\mathrm{SaO}_{2}$, procedure time, number of nodal stations, number of aspirations, medications, number of coughs and VAS scores) were compared between the two groups using the $t$ test or Wilcoxon rank-sum test, as appropriate.

A p value of $<0.05$ was considered statistically significant. All statistical analyses were performed using Stata statistical software V.9.0 (Stata Corp; College Station, Texas, USA).

\section{RESULTS}

\section{Baseline characteristics}

Consecutive patients with histologically confirmed or strongly suspected NSCLC were assessed for eligibility $(n=822)$ between June 2011 and February 2012. One hundred and sixty-two patients were randomised, 82 to Group A and 80 to Group B (figure 1). Two patients in Group A were excluded after enrolment, leaving 80 patients allocated to each group. The baseline characteristics of patients in the two groups are shown in table 1 . There were no differences between groups in age, gender, final diagnosis, location of tumours, clinical staging, $\mathrm{FEV}_{1}$, baseline blood pressure, heart rate or $\mathrm{SaO}_{2}$. 
Table 1 Clinical characteristics of enrolled patients

\begin{tabular}{|c|c|c|c|}
\hline & $\begin{array}{l}\text { Group A: } \\
\text { EBUS-centred } \\
(\mathrm{N}=80)\end{array}$ & $\begin{array}{l}\text { Group B: } \\
\text { EUS-centred } \\
(\mathrm{N}=80)\end{array}$ & $\begin{array}{l}p \\
\text { Value }\end{array}$ \\
\hline Age, mean (SD), years & $63.21(7.91)$ & $62.94(8.39)$ & 0.831 \\
\hline \multicolumn{4}{|l|}{ Gender, n (\%) } \\
\hline Men & $63(78.8)$ & $57(71.3)$ & 0.273 \\
\hline Women & $17(21.3)$ & $23(28.8)$ & \\
\hline \multicolumn{4}{|l|}{ Final diagnosis, n (\%) } \\
\hline NSCLC & $77(96.25)$ & $74(92.5)$ & 0.330 \\
\hline Squamous cell carcinoma & $28(35.0)$ & $26(32.5)$ & \\
\hline Adenocarcinoma & $41(51.3)$ & $45(56.3)$ & \\
\hline LCNEC & $2(2.5)$ & $2(2.5)$ & \\
\hline Sarcomatoid carcinoma & $1(1.25)$ & 0 & \\
\hline $\begin{array}{l}\text { Combined LCNEC and } \\
\text { adenocarcinoma }\end{array}$ & 0 & $1(1.3)$ & \\
\hline NSCLC, not specified & $5(6.3)$ & 0 & \\
\hline Small cell carcinoma & $1(1.2)$ & $2(2.5)$ & \\
\hline $\begin{array}{l}\text { Poorly differentiated } \\
\text { carcinoma }\end{array}$ & 0 & $1(1.3)$ & \\
\hline Metastatic thyroid cancer & 0 & $1(1.3)$ & \\
\hline Benign lesions & $2(2.5)$ & $2(2.5)$ & \\
\hline \multicolumn{4}{|l|}{ Location of tumours, $\mathrm{n}(\%)$} \\
\hline Right & $48(60.0)$ & $46(57.5)$ & 0.532 \\
\hline Right upper lobe & $27(33.8)$ & $20(25.0)$ & \\
\hline Right middle lobe & $7(8.8)$ & $8(10.0)$ & \\
\hline Right lower lobe & $14(17.5)$ & $18(22.5)$ & \\
\hline Left & $32(40.0)$ & $34(42.5)$ & \\
\hline Left upper lobe & $19(23.8)$ & $25(31.3)$ & \\
\hline Left lower lobe & $13(16.3)$ & $9(11.3)$ & \\
\hline \multicolumn{4}{|c|}{ Clinical T staging by chest $\mathrm{CT}, \mathrm{n}(\%)$} \\
\hline $\mathrm{T} 1$ & $26(32.50)$ & $30(37.50)$ & 0.184 \\
\hline $\mathrm{T} 2$ & $38(47.50)$ & $29(36.25)$ & \\
\hline $\mathrm{T} 3$ & $14(17.50)$ & $21(26.25)$ & \\
\hline T4 & $2(2.50)$ & 0 & \\
\hline \multicolumn{4}{|c|}{ Clinical $\mathrm{N}$ staging by PET/CT, n (\%) } \\
\hline NO & $28(35.00)$ & $28(35.0)$ & 0.860 \\
\hline N1 & $9(11.25)$ & $9(11.3)$ & \\
\hline N2 & $26(32.50)$ & $22(27.5)$ & \\
\hline N3 & $17(21.25)$ & $21(26 . .3)$ & \\
\hline Baseline $\mathrm{FEV}_{1}$, mean $(\mathrm{SD}), \mathrm{L}$ & $2.39(0.61)$ & $2.33(0.64)$ & 0.524 \\
\hline \% predicted, mean (SD), \% & $93.5(20.5)$ & $94.1(19.6)$ & 0.844 \\
\hline $\begin{array}{l}\text { Baseline systolic BP, mean } \\
\text { (SD), mm Hg }\end{array}$ & $143.2(20.5)$ & $142.7(22.2)$ & 0.878 \\
\hline $\begin{array}{l}\text { Baseline diastolic BP, mean } \\
\text { (SD), mm Hg }\end{array}$ & $81.5(12.4)$ & $80.8(10.8)$ & 0.712 \\
\hline $\begin{array}{l}\text { Baseline heart rate, mean } \\
\text { (SD), n/min }\end{array}$ & $74.4(5.8)$ & $78.0(16.5)$ & 0.159 \\
\hline Baseline $\mathrm{SaO}_{2}$, mean (SD), \% & $99.7(0.8)$ & $99.8(0.6)$ & 0.157 \\
\hline
\end{tabular}

$B P$, blood pressure; EBUS, endobronchial ultrasound; EUS, endoscopic ultrasound; $\mathrm{FEV}_{1}$, forced expiratory volume in $1 \mathrm{~s}$; LCNEC, large cell neuroendocrine carcinoma; NSCLC, non-small cell carcinoma; PET, positron emission tomography; $\mathrm{SaO}_{2}$, oxygen saturation.

\section{Results of endoscopic procedures and surgery}

The clinical course of the enrolled patients is shown in figure 1. The results of sampled nodal stations are presented in online supplementary table 2. In Group A, 239 nodal stations were sampled by EBUS-TBNA. Mediastinal metastases were diagnosed in 28 patients $(8 \mathrm{~N} 3,8$ multi-station $\mathrm{N} 2,12$ single station $\mathrm{N} 2$ ) in 54 nodal stations. With EUS-FNA, 71 nodal stations were sampled and 16 were positive for metastasis. Among them, 10 were also diagnosed as metastatic by EBUS-TBNA. Thus, EUS-FNA diagnosed six additional metastatic nodes (1 at station 1R, 2 at station 4L, 1 at station 5 and 2 at station 9). Additional gain of EUS-FNA in mediastinal staging was found in two cases. In one patient with squamous cell carcinoma at the right lower lobe, EUS-FNA upstaged from N2 to N3 by detecting metastasis at $4 \mathrm{~L}$ station. A gain with the addition of EUS-FNA in detecting mediastinal metastasis was observed in one patient with adenocarcinoma in the left lower lobe; EUS-FNA diagnosed metastasis at $4 \mathrm{~L}$ which EBUS-TBNA failed to diagnose. Among 51 patients in Group A for whom no mediastinal involvement was observed by endoscopic staging, six were not able to be evaluated for diagnostic values (figure 1). Forty-five patients underwent surgery and mediastinal lymph node dissection, which confirmed single station $\mathrm{N} 2$ disease in five patients (three at station 4R, two at station 7).

In Group B, 153 nodal stations were sampled by EUS-FNA. Metastases were diagnosed in 15 patients ( 3 N3, 4 multi-station $\mathrm{N} 2,8$ single station $\mathrm{N} 2$ ) in 22 nodal stations. EBUS-TBNA was performed on 200 nodal stations and diagnosed metastasis at 37 locations (11 already diagnosed by EUS). Among 26 metastatic nodes diagnosed only by EBUS-TBNA ( 7 at station $2 \mathrm{R}, 15$ at station $4 \mathrm{R}, 4$ at station 7), 22 locations at $2 \mathrm{R}$ and $4 \mathrm{R}$ were not accessible by EUS-FNA. Metastases at station 7 were diagnosed only by EBUS-TBNA in four patients (EUS-FNA was negative in one patient, subcarinal nodes were not well visualised by EUS in two patients, and one patient had a mediastinal haematoma following EUS-FNA). An additional benefit of EBUS-TBNA in $\mathrm{N}$ staging was found in 11 cases (figure 2). Three patients with left-sided tumours were upstaged from N2 to N3. Diagnostic gain in detecting mediastinal metastasis was obtained in eight patients with right-sided tumours (all N2). Among 57 patients in Group B for whom no mediastinal involvement was observed by endoscopic staging, six were not evaluable (figure 1). Fifty-one patients underwent surgery and mediastinal nodal dissection, which confirmed N2 disease in two patients (one multistation $\mathrm{N} 2$ at $2 \mathrm{R}$ and 7 , one single station N2 at 4R). The prevalence of mediastinal metastasis was not statistically different between Groups A and B (45.9\% (34/74) and 33.8\% (25/74), respectively; $\mathrm{p}=0.131$ ).

\section{Diagnostic values of endoscopic procedures}

The diagnostic values of procedures in detecting mediastinal metastasis $(\mathrm{N} 2$ or $\mathrm{N} 3)$ are presented in table 2. Accuracy was 93.2\% (95\% CI $87.5 \%$ to $99.0 \%$ ) and $97.3 \%$ (95\% CI $93.6 \%$ to $101.0 \%$ ) for Groups A and B, respectively, which showed no statistical difference between them $(p=0.245)$. Sensitivity and NPV were not different between groups. The sensitivity of EBUS-TBNA in Group A tended to be higher than the sensitivity of EUS-FNA in Group B (82.4\% (95\% CI $65.5 \%$ to $93.2 \%)$ and $60.0 \%$ (95\% CI $38.7 \%$ to $78.9 \%$ ), respectively), although the difference was not significant $(p=0.056)$. In Group B, adding a secondary procedure significantly increased the diagnostic accuracy $(p=0.016)$, sensitivity $(p=0.008)$ and NPV $(p=0.029)$ in detecting mediastinal metastasis, the features of which were not observed in Group A. An increase in diagnostic values by adding a secondary procedure was significantly higher in Group B than in Group A (accuracy 10.8\% (95\% CI 8.5\% to $13.1 \%)$ vs $1.4 \%$ (95\% CI $1.0 \%$ to $1.7 \%), p=0.016$; sensitivity 
A

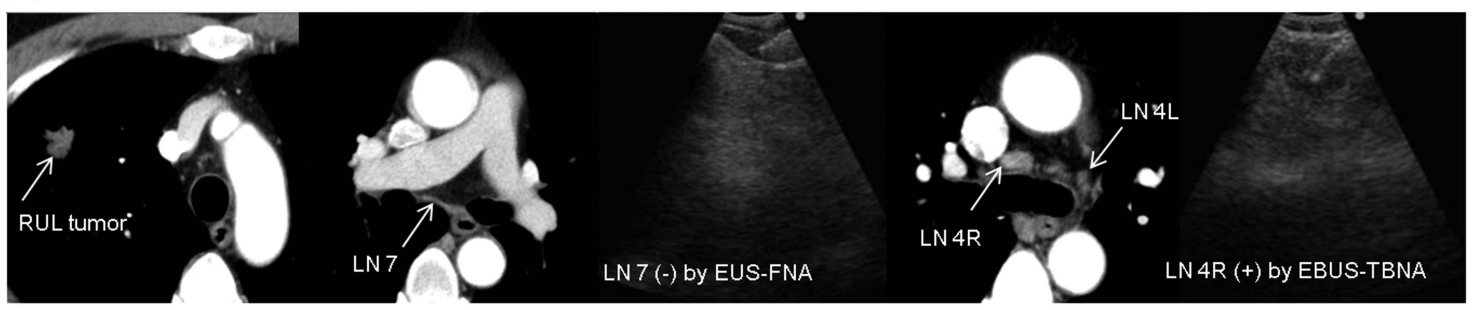

B

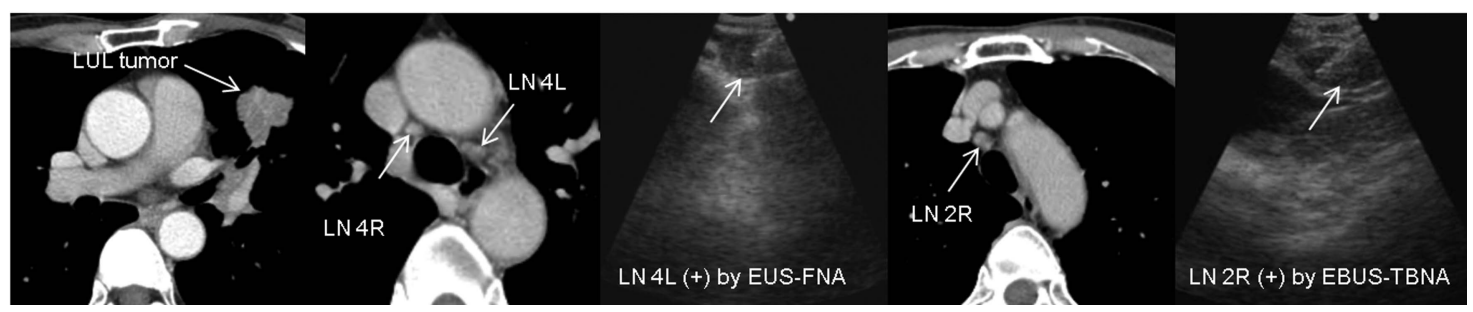

Figure 2 Representative cases with additional diagnostic benefits obtained by endobronchial ultrasound-guided transbronchial needle aspiration (EBUS-TBNA) in Group B. (A) In a patient (M/50) with RUL adenocarcinoma, endoscopic ultrasound-guided fine needle aspiration (EUS-FNA) on station 7 was negative. EBUS-TBNA was performed following EUS on stations $2 \mathrm{R}, 4 \mathrm{R}, 4 \mathrm{~L}$ and 7 as well. Station $4 \mathrm{~L}$ and the right side of the subcarinal node were not visualised well with EUS. EBUS-TBNA diagnosed N2 disease by detecting metastasis at station 4R. (B) EUS-FNA was performed on stations $4 \mathrm{~L}$ and 7 in a patient (M/69) with $\mathrm{LUL}$ adenocarcinoma. N2 disease was diagnosed by detecting metastasis at station $4 \mathrm{~L}$. EBUS-TBNA was added on station $2 \mathrm{R}$ and $4 \mathrm{R}$. N3 disease was confirmed by detecting metastasis at station 2R with EBUS-TBNA. LN, lymph node; LUL, left upper lobe; RUL, right upper lobe.

$32.0 \%$ (95\% CI $21.7 \%$ to $42.3 \%$ ) vs $2.9 \%$ (95\% CI $2.0 \%$ to $3.9 \%), p=0.002$ ). Additional diagnostic benefits in mediastinal staging (including upstaging from $\mathrm{N} 2$ to $\mathrm{N} 3$ ) were more prevalent in Group B than in Group A (11/74, 14.9\% (95\% CI 6.8\% to $23.0 \%$ ) vs $2 / 74,2.7 \% 95 \%$ CI $0.0 \%$ to $6.4 \%$ ), $\mathrm{p}=0.009$ ).

\section{Other outcomes}

Other secondary outcomes are listed in tables 3 and 4. Procedure time, number of nodal stations sampled and the number of aspirations were not different between groups. Procedure time and numbers of nodal stations and aspirations were different between groups when assessed by EBUS and EUS separately. The amount of medications used during the procedures was similar between the groups. Mean systolic blood pressure (SBP), maximum SBP and mean $\mathrm{SaO}_{2}$ were not different between groups. Changes in these cardiorespiratory parameters from baseline were not statistically different between groups (data not shown). Cardiorespiratory parameters during the first procedure were similar between groups except mean $\mathrm{SaO}_{2}$ that showed small difference $(0.7 \%)$ in mean values. Mean SBP, maximum SBP and mean heart rate in EBUS time were lower in Group A, and mean SBP and mean heart rate of EUS time was lower in Group B. $\mathrm{SaO}_{2}$ in EUS time was higher in Group B, although the difference was small. The number of coughs counted during the procedure and the number of patients with hypoxia or irritable movements were also similar. In Group B, pneumomediastinum was observed during the EBUS procedure in one patient and resolved without specific treatment. No other major complications were observed. The minor complications observed are shown in table 3; no intergroup differences were observed.

There was no difference in procedure difficulty as assessed by the bronchoscopist and nurse assistant. In patient satisfaction parameters, we observed similar results between the groups in overall procedure tolerance, tolerance of cough, dyspnoea, pain and nausea assessed several hours and several days after the

Table 2 Diagnostic values of procedures in the detection of mediastinal metastasis

\begin{tabular}{llll}
\hline & $\begin{array}{l}\text { Group A: EBUS-centred } \\
(\mathbf{n}=74)\end{array}$ & $\begin{array}{l}\text { Group B: EUS-centred } \\
(\mathbf{n}=74)\end{array}$ & $\mathbf{p}$ Value \\
\hline Diagnostic accuracy, \% $(\mathrm{n} / \mathrm{n})$ & $93.2(69 / 74)(87.5$ to 99.0$)$ & $97.3(72 / 74)(93.6$ to 101.0$)$ & $86.5(64 / 74)(78.7$ to 94.3$)$ \\
Accuracy of first procedure*, \% $(\mathrm{n} / \mathrm{n})$ & $91.9(68 / 74)(85.7$ to 98.1$)$ & 0.016 & 0.245 \\
$\mathrm{p}$ Value & 0.754 & $92.0(23 / 25)(74.0$ to 99.0$)$ & 0.290 \\
Sensitivity, \% $(\mathrm{n} / \mathrm{n})$ & $85.3(29 / 34)(68.9$ to 95.0$)$ & $60.0(15 / 25)(38.7$ to 78.9$)$ & 0.008 \\
Sensitivity of first procedure, \% $(\mathrm{n} / \mathrm{n})$ & $82.4(28 / 34)(65.5$ to 93.2$)$ & $96.1(49 / 51)(86.5$ to 99.5$)$ \\
$\mathrm{p}$ Value & 0.742 & $83.1(49 / 59)(71.0$ to 91.6$)$ \\
NPV, \% $(\mathrm{n} / \mathrm{n})$ & $88.9(40 / 45)(75.9$ to 96.3$)$ & 0.029 & 0.056 \\
NPV of first procedure, \% $(\mathrm{n} / \mathrm{n})$ & $87.0(40 / 46)(73.7$ to 95.1$)$ & 0.581 \\
$\mathrm{p}$ Value & 0.777 & & \\
\hline
\end{tabular}

Data shown as $(\mathrm{n} / \mathrm{n})(95 \% \mathrm{Cl})$.

*First procedure: EBUS in Group A; EUS in Group B.

EBUS, endobronchial ultrasound; EUS, endoscopic ultrasound; NPV, negative predictive value. 
Table 3 Secondary outcomes measured during procedures, medications and complications

\begin{tabular}{|c|c|c|c|}
\hline & $\begin{array}{l}\text { Group A: EBUS-centred } \\
(\mathrm{n}=80)\end{array}$ & $\begin{array}{l}\text { Group B: EUS-centred } \\
(n=80)\end{array}$ & $\mathrm{p}$ Value \\
\hline Total procedure time, mean (SD), min:s & $38: 3(7: 13)$ & $39: 4(9: 30)$ & 0.422 \\
\hline EBUS procedure time, mean (SD), min:s & 23:5 (5:39) & $18: 1(6: 6)$ & $<0.0001$ \\
\hline EUS procedure time, mean (SD), min:s & $6: 4(5: 15)$ & $13: 5(8: 3)$ & $<0.0001$ \\
\hline No. of nodal stations, mean (SD) & $3.5(0.9)$ & $3.7(1.2)$ & 0.232 \\
\hline No. nodal stations by EBUS-TBNA, mean (SD) & $3.0(0.8)$ & $2.5(0.8)$ & 0.000 \\
\hline No. nodal stations by EUS-FNA, mean (SD) & $0.9(0.9)$ & $1.9(1.2)$ & $<0.0001$ \\
\hline No. of aspirations, mean (SD) & $9.6(2.9)$ & $10.2(3.6)$ & 0.320 \\
\hline No. of aspirations by EBUS-TBNA, mean (SD) & $8.1(2.4)$ & $6.1(2.4)$ & $<0.0001$ \\
\hline No. of aspirations by EUS-FNA, mean (SD) & $1.6(1.8)$ & $4.1(2.8)$ & $<0.0001$ \\
\hline Midazolam, mean (SD), mg & $3.0(0.6)$ & $3.0(0.6)$ & 0.670 \\
\hline Fentanyl, mean (SD), $\mu \mathrm{g}$ & $50.8(10.6)$ & $51.7(7.4)$ & 0.809 \\
\hline Lidocaine, mean (SD), mg & $442.3(61.2)$ & $431.3(53.4)$ & 0.228 \\
\hline Mean SBP, mean (SD), mm Hg & $144.2(20.1)$ & $145.9(22.8)$ & 0.629 \\
\hline Mean SBP during first procedure, mean (SD), $\mathrm{mm} \mathrm{Hg}$ & $138.8(22.8)$ & $141.1(22.2)$ & 0.512 \\
\hline Mean SBP during EBUS time, mean (SD), mm Hg & $138.8(22.8)$ & $151.4(25.5)$ & 0.001 \\
\hline Mean SBP during EUS time, mean (SD), mm Hg & $149.6(25.4)$ & $141.1(22.2)$ & 0.026 \\
\hline Maximum SBP, mean (SD), mm Hg & $171.1(27.4)$ & $174.9(31.0)$ & 0.422 \\
\hline Maximum SBP during first procedure, mean (SD), $\mathrm{mm} \mathrm{Hg}$ & $157.4(27.5)$ & $151.2(28.1)$ & 0.160 \\
\hline Maximum SBP during EBUS time, mean (SD), mm Hg & $157.4(27.5)$ & $170.7(30.8)$ & 0.004 \\
\hline Maximum SBP during EUS time, mean (SD), mm Hg & $156.7(28.4)$ & $151.2(28.1)$ & 0.213 \\
\hline Mean $H R$, mean (SD), n/min & $82.9(16.0)$ & $82.6(12.5)$ & 0.891 \\
\hline Mean HR during first procedure, mean (SD), n/min & $80.0(18.8)$ & $77.4(13.2)$ & 0.314 \\
\hline Mean HR during EBUS time, mean (SD), n/min & $80.0(18.8)$ & $88.8(18.5)$ & 0.003 \\
\hline Mean HR during EUS time, mean (SD), n/min & $84.5(17.0)$ & $77.4(13.2)$ & 0.003 \\
\hline Mean $\mathrm{SaO}_{2}$, mean (SD), \% & $97.7(1.5)$ & $98.3(2.4)$ & 0.084 \\
\hline Mean $\mathrm{SaO}_{2}$ during first procedure, mean (SD), \% & $98.1(1.6)$ & $98.8(1.2)$ & 0.002 \\
\hline Mean $\mathrm{SaO}_{2}$ during EBUS time, mean (SD), \% & $98.1(1.6)$ & $97.9(1.6)$ & 0.590 \\
\hline Mean $\mathrm{SaO}_{2}$ during EUS time, mean (SD), \% & $97.9(1.9)$ & $98.8(1.2)$ & 0.001 \\
\hline Number of coughs, median (IQR) & $95.5(100)$ & $89.5(115.5)$ & 0.921 \\
\hline Coughs during EBUS time, median (IQR) & $40.5(87)$ & $58.5(86)$ & 0.226 \\
\hline Coughs during EUS time, median (IQR) & $6(14)$ & $5(15)$ & 0.434 \\
\hline Hypoxic event, no. of patients & 17 & 19 & 0.705 \\
\hline Irritable movement, no of patients & 3 & 2 & 1.000 \\
\hline \multicolumn{4}{|l|}{ Major complications } \\
\hline Pneumomediastinum & 0 & 1 & 1.000 \\
\hline \multicolumn{4}{|l|}{ Minor complications } \\
\hline Mediastinal or perinodal bleeding & 5 & 7 & 0.548 \\
\hline Bleeding by EBUS-TBNA & 3 & 0 & 0.245 \\
\hline Bleeding by EUS-FNA & 2 & 7 & 0.167 \\
\hline Hiccup after procedure & 2 & 1 & 1.000 \\
\hline Chest pain after procedure & 0 & 1 & 1.000 \\
\hline
\end{tabular}

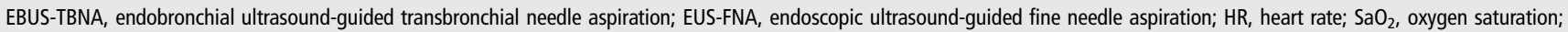
SBP, systolic blood pressure.

procedures. The proportion of patients not willing to return if a repeat procedure was indicated did not differ between the groups (table 4).

\section{DISCUSSION}

To our knowledge, this is the first randomised controlled study to evaluate the impact of procedure sequence in the combined approach of EBUS and EUS in mediastinal staging of lung cancer. Our results showed that overall diagnostic accuracy was similar between EBUS-centred and EUS-centred procedures. This similarity was expected because secondary procedures were added after primary procedures to cover inaccessible locations. Additional gains by performing secondary procedures were significantly higher in Group B. We observed that diagnostic accuracy, sensitivity and NPV increased by adding EBUS in Group
B. However, in the EBUS-centred group, we did not observe a significant increase in diagnostic values by adding EUS, as EUS detected only one additional case with mediastinal metastasis following EBUS.

We believe the differences in additional diagnostic benefits by adding secondary procedures are based on the differences in accessibility of EBUS and EUS to the mediastinum. ${ }^{7} 8{ }^{8} 13$ EUS-FNA has a limitation in targeting pretracheal lesions that are easily accessed by EBUS-TBNA. Nodal stations $2 \mathrm{R}$ and $4 \mathrm{R}$ were generally inaccessible by EUS-FNA in patients with potentially operable lung cancer with relatively small mediastinal nodes. ${ }^{13}$ In the present study, additional diagnostic benefits of EBUS-TBNA in the EUS-centred group were observed by targeting nodal station $2 \mathrm{R}, 4 \mathrm{R}$ and some subcarinal nodes that were inaccessible or difficult to access by EUS. EUS-FNA 
Table 4 Procedure tolerance parameters measured after procedures

\begin{tabular}{|c|c|c|c|}
\hline & $\begin{array}{l}\text { Group A: } \\
\text { EBUS-centred } \\
(\mathrm{n}=80)\end{array}$ & $\begin{array}{l}\text { Group B: } \\
\text { EUS-centred } \\
(\mathrm{n}=80)\end{array}$ & p Value \\
\hline $\begin{array}{l}\text { Procedure difficulty, bronchoscopist, } \\
\text { VAS score, mean (SD) }\end{array}$ & $4.56(1.36)$ & $4.60(1.33)$ & 0.838 \\
\hline $\begin{array}{l}\text { Procedure difficulty, assisting nurse, } \\
\text { VAS score, mean (SD) }\end{array}$ & $4.96(1.39)$ & $4.97(1.59)$ & 0.958 \\
\hline \multicolumn{4}{|c|}{ Patient satisfaction parameters assessed after procedure $(2-4 \mathrm{~h})$} \\
\hline Overall tolerance, VAS score, mean (SD) & $4.03(2.51)$ & $4.38(2.28)$ & 0.350 \\
\hline Cough, VAS score, mean (SD) & $3.13(3.10)$ & $3.61(2.92)$ & 0.313 \\
\hline Dyspnoea, VAS score, mean (SD) & $1.72(2.56)$ & $2.04(2.88)$ & 0.461 \\
\hline Pain, VAS score, mean (SD) & $2.10(2.87)$ & $1.94(2.81)$ & 0.727 \\
\hline Nausea, VAS score, mean (SD) & $0.96(2.05)$ & $1.18(2.28)$ & 0.524 \\
\hline Not willing to repeat, $\mathrm{n}(\%)$ & $7(8.8)$ & $5(6.3)$ & 0.548 \\
\hline \multicolumn{4}{|c|}{ Patient satisfaction parameters, assessed after procedure (3-5 days) } \\
\hline Overall tolerance, VAS score, mean (SD) & $4.06(2.43)$ & $4.60(2.36)$ & 0.152 \\
\hline Cough, VAS score, mean (SD) & $2.69(2.84)$ & $3.07(3.10)$ & 0.415 \\
\hline Dyspnoea, VAS score, mean (SD) & $1.94(2.64)$ & $2.03(2.82)$ & 0.851 \\
\hline Pain, VAS score, mean (SD) & $2.24(3.00)$ & $2.51(3.10)$ & 0.569 \\
\hline Nausea, VAS score, mean (SD) & $0.58(1.60)$ & $0.67(1.80)$ & 0.746 \\
\hline Not willing to repeat, $\mathrm{n}(\%)$ & $5(6.3)$ & $7(8.8)$ & 0.548 \\
\hline
\end{tabular}

has an advantage in targeting paraoesophageal, pulmonary ligament and some subaortic nodes. However, in our previous study the proportion of these nodal stations accessible only by EUS was not high (6.1\%) in patients with operable lung cancer. ${ }^{13}$ When mediastinal nodal stations having at least one node $\geq 5 \mathrm{~mm}$ were evaluated in operable lung cancer, accessibility to the mediastinum was $78.6 \%$ and $50.7 \%$ by EBUS-TBNA and EUS-FNA, respectively. ${ }^{13}$

High accessibility to the mediastinum would result in high diagnostic values in detecting mediastinal metastasis. In our study, the sensitivity of the first procedure tended to be higher in the EBUS-centred group than in the EUS-centred group $(82.4 \%$ vs $60.0 \%)$, although the differences were not statistically significant in the small group of patients. High diagnostic values of EBUS-TBNA in lung cancer staging have been reported. According to meta-analyses, pooled sensitivities of EBUS-TBNA in diagnosing mediastinal metastasis were $88-93 \% .^{1-3}$ In patients with potentially operable lung cancer, the sensitivity of EBUS-TBNA was reported as 81-92\%. ${ }^{5} 13$ Yasufuku et al showed comparable sensitivity of EBUS-TBNA with mediastinoscopy in operable lung cancer. ${ }^{5}$ The role of EUS-FNA in lung cancer staging has been studied and the pooled sensitivities were reported as $83-89 \%$ in meta-analyses. ${ }^{14}$ EUS-FNA had a sensitivity of $63-76 \%$ in patients with potentially operable lung cancer. ${ }^{18-20}$ Although many studies have been performed on the role of EBUS-TBNA or EUS-FNA in lung cancer staging, a direct comparison of EBUS and EUS has not been well studied in potentially operable patients. Wallace et al compared EBUS-TBNA and EUS-FNA in detecting mediastinal metastasis in 138 patients with suspected lung cancer. Operability was not included in the enrolment criteria; diagnostic sensitivity was the same for the two procedures (69\%). ${ }^{11}$

Given the high diagnostic yields of EBUS-TBNA and the small additional benefits of EUS-FNA following EBUS in mediastinal staging, performing EBUS-TBNA as a primary procedure may be the best option. However, EUS-FNA is generally well tolerated, and procedure tolerance may affect the selection procedure. In our study, however, we did not observe any differences in procedure time, cardiorespiratory parameters, complications, procedure difficulty or patient satisfaction between the groups. Similar cardiorespiratory parameters and patient satisfaction could be due to the fact that the procedures were performed under sedation. SBP and heart rate during the first procedure were also similar between groups, demonstrating that EBUS and EUS procedures result in similar haemodynamic effects when performed under sedation in patients with operable lung cancer. Similar procedure times, cardiorespiratory parameters and patient satisfaction may also be related to the high requirement of EBUS and relatively long procedure time following EUS in Group B. SBP, heart rate and $\mathrm{SaO}_{2}$ tended to be better when EBUS (or EUS) was used as the first procedure than when either was used as the second procedure. This could be related to a decreased level of sedation at the end of the procedures.

The location of tumours and target lymph nodes influences procedure selection. Because a combination of EBUS and EUS in lung cancer staging increases medical costs, a single procedure is usually selected. In this study we applied stratified randomisation according to tumour location to avoid tumour location bias. In Group B an additional gain was observed primarily in patients with right-sided tumours. However, we also identified patients with left side tumours who were diagnosed with N3 disease by adding EBUS in Group B. EUS may be the preferable procedure in patients with left-sided tumours, however, adding EBUS following EUS is still reasonable to access the right side mediastinum. The location of lymph nodes suspected of malignancy in imaging studies can guide procedure selection. However, mediastinal metastasis is observed even in patients with negative findings on CT or positron emission tomography (PET). ${ }^{21} 22$ Suspicion raised by imaging tools alone can be insufficient.

The high diagnostic yield of EBUS-TBNA, small additional benefit of EUS and similar patient satisfaction between the two groups suggest that an EBUS-centred procedure or EBUS alone is preferable in mediastinal staging of lung cancer. However, in this study we used EUS-FNA in patients with operable lung cancer with tolerable cardiorespiratory function. EUS can be a better procedure in patients with poor cardiorespiratory function. ${ }^{15}$ Another advantage of EUS-FNA is that left adrenal gland and coeliac lymph nodes can be examined through the stomach, ${ }^{23} 24$ although we did not use an ultrasound bronchoscope for these purposes in this study. When using EUS-FNA for diagnosis of a specific location accessible by EUS, EUS-FNA alone is a good diagnostic method. ${ }^{25-27}$

Our study has several limitations. We used EUS-FNA with an ultrasound bronchoscope instead of conventional EUS-FNA. Disadvantages of EUS-FNA with an ultrasound bronchoscope include a limited overview due to a narrow sonographic angle, fixed needle angle and limited ability to push the oesophageal wall when using a thin bronchoscope, although accessibility of this procedure seemed similar to conventional EUS. ${ }^{13} 15$ Another limitation is that this study was performed in a single institution. Diagnostic yields of procedures would be more affected by the experience of a single institution compared with large multicentre trials.

In conclusion, there were no differences in diagnostic values and patient satisfaction between the EBUS-centred group and the EUS-centred group. However, considering the requirement of EBUS following EUS, EBUS-TBNA is the preferable primary procedure to consider for endoscopic mediastinal staging of potentially operable lung cancer. 
Acknowledgements We thank all patients, physicians, clinical trial nurses, laboratory staff and technicians at the Center for Lung Cancer, National Cancer Center, Goyang, Korea.

Contributors HJK: Data analysis and interpretation, manuscript writing and final approval of manuscript. BH: Concept and design, provision of study patients, endoscopic procedures, collection and assembly of data, data analysis and interpretation, manuscript writing and final approval of manuscript. G-KL: Pathologic interpretation and final approval of manuscript. B-HN: Statistical analysis and final approval of manuscript. H-SL, MSK, JML, JIZ: Provision of study patients, surgical staging and final approval of manuscript. HSL, J-YH: Provision of study patients and final approval of manuscript.

Funding This work was supported by National Cancer Center Grant 1110570. The study sponsor had no role in the study design, collection, analysis and interpretation of data, writing of the report and in the decision to submit the paper for publication.

\section{Competing interests None}

Ethics approval This study is the NCCCTS 11-539 trial performed at the National Cancer Center, Korea. The ethical review committee of our institution approved the protocol for this study.

Patient consent Obtained.

Provenance and peer review Not commissioned, internally peer reviewed.

\section{REFERENCES}

1 Silvestri GA, Gonzalez AV, Jantz MA, et al. Methods for staging non-small cell lung cancer: diagnosis and management of lung cancer, 3rd ed: American College of Chest Physicians evidence-based clinical practice guidelines. Chest 2013;143:e211S-50S.

2 Adams K, Shah PL, Edmonds L, et al. Test performance of endobronchial ultrasound and transbronchial needle aspiration biopsy for mediastinal staging in patients with lung cancer: systematic review and meta-analysis. Thorax 2009;64:757-62.

3 Gu P, Zhao YZ, Jiang LY, et al. Endobronchial ultrasound-guided transbronchia needle aspiration for staging of lung cancer: a systematic review and meta-analysis. Eur J Cancer 2009:45:1389-96.

4 Micames CG, McCrory DC, Pavey DA, et al. Endoscopic ultrasound-guided fine-needle aspiration for non-small cell lung cancer staging: a systematic review and metaanalysis. Chest 2007;131:539-48.

5 Yasufuku K, Pierre T, Darling G, et al. A prospective controlled trial of endobronchial ultrasound-guided transbronchial needle aspiration compared with mediastinoscopy for mediastinal lymph node staging of lung cancer. J Thorac Cardiovasc Surg 2011:142:1393-400.

6 Annema JT, van Meerbeeck JP, Rintoul RC, et al. Mediastinoscopy vs endosonography for mediastinal nodal staging of lung cancer: a randomized trial. JAMA 2010;304:2245-52

7 Herth FJ, Rabe KF, Gasparini S, et al. Transbronchial and transoesophagea (ultrasound-guided) needle aspirations for the analysis of mediastinal lesions. Eur Respir J 2006:28:1264-75.

8 Yasufuku K, Nakajima T, Chiyo M, et al. Endobronchial ultrasonography: current status and future directions. J Thorac Oncol 2007;2:970-9.

9 Rintoul RC, Skwarski KM, Murchison JT, et al. Endobronchial and endoscopic ultrasound-guided real-time fine-needle aspiration for mediastinal staging. Eur Respir I 2005:25:416-21.

10 Vilmann P, Krasnik M, Larsen SS, et al. Transesophageal endoscopic ultrasound-guided fine-needle aspiration (EUS-FNA) and endobronchial ultrasound-guided transbronchial needle aspiration (EBUS-TBNA) biopsy: a combined approach in the evaluation of mediastinal lesions. Endoscopy 2005;37:833-9

11 Wallace MB, Pascual JM, Raimondo $M$, et al. Minimally invasive endoscopic staging of suspected lung cancer. JAMA 2008;299:540-6.

12 Herth FJ, Krasnik M, Kahn N, et al. Combined endoscopic-endobronchial ultrasound-guided fine-needle aspiration of mediastinal lymph nodes through a single bronchoscope in 150 patients with suspected lung cancer. Chest 2010:138:790-4

13 Hwangbo B, Lee GK, Lee HS, et al. Transbronchial and transesophageal fine-needle aspiration using an ultrasound bronchoscope in mediastinal staging of potentially operable lung cancer. Chest 2010;138:795-802.

14 Annema JT, Rabe KF. Endosonography for lung cancer staging: one scope fits all? Chest 2010;138:765-7.

15 Hwangbo B, Lee HS, Lee GK, et al. Transoesophageal needle aspiration using a convex probe ultrasonic bronchoscope. Respirology 2009;14:843-9.

16 Fleiss JL, Tytun A, Ury HK. A simple approximation for calculating sample sizes for comparing independent proportions. Biometrics 1980;36:343-6.

17 Yasufuku K, Nakajima T, Motoori K, et al. Comparison of endobronchial ultrasound, positron emission tomography, and CT for lymph node staging of lung cancer. Chest 2006;130:710-18.

18 Annema JT, Versteegh MI, Veselic M, et al. Endoscopic ultrasound added to mediastinoscopy for preoperative staging of patients with lung cancer. JAMA 2005:294:931-6.

19 Talebian M, von Bartheld MB, Braun J, et al. EUS-FNA in the preoperative staging of non-small cell lung cancer. Lung Cancer 2010:69:60-5.

20 Fritscher-Ravens A, Davidson BL, Hauber HP, et al. Endoscopic ultrasound, positron emission tomography, and computerized tomography for lung cancer. Am J Respir Crit Care Med 2003;168:1293-7.

21 Herth FJ, Ernst A, Eberhardt R, et al. Endobronchial ultrasound-guided transbronchial needle aspiration of lymph nodes in the radiologically normal mediastinum. Eur Respir J 2006;28:910-14.

22 Hwangbo B, Kim SK, Lee HS, et al. Application of endobronchial ultrasoundguided transbronchial needle aspiration following integrated PET/CT in mediastinal staging of potentially operable non-small cell lung cancer. Chest 2009;135: $1280-7$.

23 Schuurbiers OC, Tournoy KG, Schoppers HJ, et al. EUS-FNA for the detection of left adrenal metastasis in patients with lung cancer. Lung Cancer 2011; 73:310-15

24 Annema JT. E(B)US-guided pulmonary interventions. Tech Gastrointest Endosc 2009;11:154-61

25 Annema JT, Veselic M, Rabe KF. EUS-guided FNA of centrally located lung tumours following a non-diagnostic bronchoscopy. Lung Cancer 2005;48:357-61; discussion 63-4.

26 Varadarajulu S, Hoffman BJ, Hawes RH, et al. EUS-guided FNA of lung masses adjacent to or abutting the esophagus after unrevealing CT-guided biopsy or bronchoscopy. Gastrointest Endosc 2004;60:293-7.

27 Caddy G, Conron M, Wright G, et al. The accuracy of EUS-FNA in assessing mediastinal lymphadenopathy and staging patients with NSCLC. Eur Respir $\int$ 2005:25:410-15

28 Goldstraw P, Crowley J, Chansky K, et al. The IASLC Lung Cancer Staging Project: proposals for the revision of the TNM stage groupings in the forthcoming (seventh) edition of the TNM Classification of malignant tumours. J Thorac Oncol 2007;2:706-14 